\title{
Programa nacional de triagem neonatal: achados em exames de recém-nascidos de uma maternidade escola
}

\author{
National neonatal screening program: findings of newborns from a maternity school \\ Programa nacional de triage neonatal: hallazgos en exámenes de recién nacidos de una maternidad escuela \\ Larissa Bento de Araújo Mendonça ${ }^{1}$ (D) https://orcid.org/0000-0001-7874-4497 \\ Francisca Elisângela Teixeira Lima ${ }^{1}$ (D) https://orcid.org/0000-0002-7543-6947 \\ Igor de Freitas ${ }^{1}$ (D) https://orcid.org/0000-0003-3813-5747 \\ Sabrina de Souza Gurgel ${ }^{1}$ (D) https://orcid.org/0000-0002-2180-5946 \\ Mayara Kelly Moura Ferreira ${ }^{1}$ (D) https://orcid.org/0000-0001-7823-1843 \\ Lilia Jannet Saldarriaga Sandoval ${ }^{1}$ (D) https://orcid.org/0000-0002-1773-6669
}

\section{Resumo}

Objetivo: Analisar os achados dos exames de triagem neonatal em recém-nascidos de um alojamento conjunto em uma maternidade escola do nordeste brasileiro.

Métodos: Estudo descritivo, transversal, quantitativo, realizado em uma maternidade escola pública. Amostra composta por 116 prontuários de recém-nascidos admitidos no alojamento conjunto da referida maternidade. Para coleta de dados utilizou-se instrumento elaborado pelos pesquisadores para pesquisas em prontuários. Os resultados foram apresentados em forma de tabelas, processados e analisados pela estatística descritiva.

Resultados: Houve prevalência dos seguintes dados: tipo de parto vaginal $(66,3 \%)$; sexo feminino $(42,2 \%)$, nascidos a termo (94,0\%); apgar com pontuação de oito a dez no primeiro e no quinto minuto e exames de triagem neonatal dentro das normalidades (teste do reflexo vermelho $(99,2 \%)$, teste de oximetria de pulso (100\%), triagem auditiva neonatal (72,4\%), avaliação do frênulo lingual (93,2\%)).

Conclusão: Conclui-se que a maioria dos exames de triagem neonatal teve resultados dentro dos parâmetros da normalidade.

\section{Abstract}

Objective: To analyze the findings of newborn screening tests in newborns from a joint accommodation in a school maternity hospital in northeastern Brazil.

Methods: Descriptive, cross-sectional, quantitative study conducted at a public school maternity hospital. Sample composed of 116 records of newborns admitted to the joint lodging of said maternity. For data collection, an instrument was developed by the researchers for research in medical records. The results were presented in tables, processed and analyzed by descriptive statistics.

Results: The following data were prevalent: type of vaginal delivery (66.3\%); female (42.2\%), born at term (94.0\%); (red reflex $(99.2 \%)$, pulse oximetry $(100 \%)$, neonatal hearing screening $(72.4 \%)$, and \%), evaluation of the lingual frenulum $(93.2 \%)$

Conclusion: It was concluded that the majority of the neonatal screening tests had results within the parameters of normality.

\section{Resumen}

Objetivo: Objetivo: analizar los resultados de las pruebas de detección de recién nacidos en recién nacidos de un alojamiento conjunto en un hospital de maternidad escolar en el noreste de Brasil.

Métodos: Estudio descriptivo, transversal, cuantitativo, realizado en una maternidad escolar pública. Una muestra compuesta por 116 prontuarios de recién nacidos admitidos en el alojamiento conjunto de la referida maternidad. Para la recolección de datos se utilizó instrumento elaborado por los investigadores para investigaciones en prontuarios. Los resultados fueron presentados en forma de tablas, procesados y analizados por la estadística descriptiva.

Resultados: Hubo prevalencia de los siguientes datos: tipo de parto vaginal $(66,3 \%)$; sexo femenino $(42,2 \%)$, nacidos a término (94,0\%); Puntuación de Apgar con ocho a diez en la primera y quinta minutos y pruebas de cribado neonatal dentro de la normalidad (prueba de reflejo rojo $(99,2 \%)$, prueba de oximetría de pulso (100\%), el cribado neonatal (72,4\%), evaluación del frénulo lingual (93,2\%).

Conclusion: Se concluye que la mayoría de los exámenes de selección neonatal tuvieron resultados dentro de los parámetros de la normalidad.

\section{Descritores}

Programa nacional de triagem neonatal; Neonatologia; Enfermagem pediátrica

\section{Keywords}

National health programs; Neonatology; Pediatric nursing

\section{Descriptores}

Programas nacionales de salud; Neonatología; Enfermería pediátrica

\section{Como citar:}

Mendonça LB, Lima FE, Freitas I, Gurgel SS, Ferreira MK, Sandoval L. [National neonatal screening program: findings of newborns from a maternity school]. Rev Soc Bras Enferm Ped. 2019;19(2):74-9. Portuguese

\footnotetext{
${ }^{1}$ Universidade Federal do Ceará, Fortaleza, CE, Brasil.

Conflitos de interesse: nada a declarar.

Submetido: 7 de Junho de 2019 | Aceito: 20 de Dezembro de 2019

Autor correspondente: Larissa Bento de Araújo Mendonça | E-mail: laraenfermagem@hotmail.com

DOI: http://dx.doi.org/10.31508/1676-3793201900010
} 


\section{Introdução}

O diagnóstico precoce, tratamento e acompanhamento médico de algumas patologias no recém-nascido (RN) podem evitar deficiências físicas e mentais e até mesmo a morte. Além disso, a intervenção precoce para tais patologias pode proporcionar uma melhor qualidade de vida, principalmente quando a doença pode comprometer o desenvolvimento normal da criança. Diante disto, criou-se no Brasil o Programa Nacional de Triagem Neonatal. ${ }^{(1,2)}$

Estimativas apontam que no ano de 2011, 84,8\% das crianças nascidas no Brasil foram submetidas à triagem neonatal. No entanto, o programa busca a cobertura de $100 \%$ dos RN vivos no país. ${ }^{(3)}$

Os testes que atualmente fazem parte do Programa Nacional de Triagem Neonatal são: teste do reflexo vermelho ou triagem oftalmológica ou teste do olhinho; teste de oximetria de pulso ou triagem cardíaca ou teste do coraçãozinho; triagem auditiva ou teste da orelhinha ou exame de emissões otoacústicas evocadas (EOE); avaliação do frênulo lingual ou teste da linguinha e teste do pezinho. ${ }^{(4)}$

Tendo em vista que os casos de cegueira são passíveis de prevenção, o que implicaria na melhora na qualidade de vida, além de redução considerável dos custos econômicos e sociais dos tratamentos especializados e programas de reabilitação, o teste do reflexo vermelho no RN é uma alternativa eficaz e barata na detecção de problemas oculares nos primeiros dias de vida do bebê. ${ }^{(5)}$

No teste do reflexo vermelho, busca-se como principal sinal clínico a leucocoria, e para a realização do mesmo utiliza-se um instrumento denominado gradiente de cores do teste do reflexo vermelho, composto por nuances de cores distribuídas em gradientes de vermelho, laranja e amarelo que ao serem direcionadas ao longo do eixo visual do espaço pupilar, refletem um brilho homogêneo de cor laranja-avermelhado, indicando que as estruturas internas do olho (córnea, cristalino e vítreo) estão transparentes, permitindo que a retina seja atingida pela luz de forma normal. (5,6) $^{\circ}$

Adota-se a classificação "normal ou presente", quando o reflexo em ambos os olhos são equivalentes na cor, intensidade, clareza e não existem opacidades ou pontos brancos em alguma área, em um ou em ambos os reflexos observados. O resultado será "anormal ou ausente" quando o oposto ao que foi mencionado for encontrado. ${ }^{(5)}$
O teste de oximetria de pulso detecta cardiopatias congênitas e deve ser realizado após $24 \mathrm{~h}$ de vida do RN. Para tanto, utiliza-se o monitor de oximetria de pulso, para verificação da saturação de oxigênio arterial do membro superior direito e de qualquer um dos membros inferiores, ${ }^{(7)}$ cujo resultado é positivo ou alterado quando a diferença entre os valores do membro superior direito e membro inferior for $>3 \%$ ou quando uma delas for $<95 \%{ }^{(8)}$

A triagem auditiva neonatal é realizada para detectar precocemente alterações auditivas que poderão interferir na qualidade de vida do indivíduo e deve ser realizada em todos os $\mathrm{RN}$, preferencialmente antes da alta hospitalar da maternidade. ${ }^{(9,10)}$

O teste é consiste na utilização de medidas eletroacústicas e eletrofisiológicas, como as emissões otoacústicas evocadas por transiente (EOE). Por meio de uma sonda introduzida no canal auditivo do RN, são emitidos estímulos acústicos de baixa intensidade em cada orelha, de forma randômica. O critério de referência para passar no teste (exame normal), é presença de resposta superior a $3 \mathrm{~dB}$ na banda de frequência de $1.500 \mathrm{~Hz}$ e superior a $6 \mathrm{~dB}$ nas bandas de frequências 2000, 3000 e $4000 \mathrm{~Hz}^{(9)}$

O Joint Committee on Infant Hearing preconiza que a triagem auditiva neonatal deve ser realizada até o primeiro mês de vida e que os bebês com exames alterados devam ser submetidos à avaliação audiológica, no máximo, até o terceiro mês de vida. No caso de confirmação de perda auditiva, a intervenção deve ter início até o sexto mês de vida. ${ }^{(10)}$

Na avaliação do frênulo lingual, permite-se diagnosticar os casos mais graves de língua presa e indicar a frenotomia lingual ainda na maternidade. É realizada nas primeiras 48 horas após o nascimento e nos casos em que houver dúvidas na avaliação ou não for possível visualizar o frênulo lingual, o RN deverá ser encaminhado para serviço especializado para realização do reteste com 30 dias de vida. ${ }^{(2,11)}$

O teste do pezinho, cuja finalidade é detectar doenças metabólicas, genéticas e/ou infecciosas, ${ }^{(12)}$ não foi incluído nessa pesquisa, tendo em vista que o mesmo só pode ser coletado a partir de $72 \mathrm{~h}$ de vida, sendo que os RN do setor recebem alta com 48 h de vida, realizando a coleta geralmente na atenção primária. E aqueles que coletam o teste do pezinho ainda na instituição, só recebem o resultado do exame com 45 dias. 
Dada a importância da triagem neonatal no RN para a identificação de patologias e tratamento precoce e da pouca literatura existente que abordem tais exames, evidenciando assim lacunas no conhecimento sobre a temática, teve-se como objetivo do estudo: Analisar os achados dos exames de triagem neonatal em recém-nascidos de um alojamento conjunto em uma maternidade escola do nordeste brasileiro.

\section{Métodos}

Estudo descritivo, transversal, de natureza quantitativa realizado no alojamento conjunto de uma maternidade escola do nordeste brasileiro.

A população do estudo foi composta por 219 prontuários de $\mathrm{RN}$ admitidos na referida unidade, provenientes da sala de parto durante o mês de maio de 2017. Adotou-se o período de um mês para a composição da amostra tendo em vista o alto índice de admissões no setor, fornecendo um quantitativo satisfatório para a análise dos dados pelos pesquisadores. A amostra foi composta por prontuários de RN que atenderem aos seguintes critérios de inclusão: prontuários de RN admitido no mês de maio de 2017; disponível no momento da coleta de dados e que tivessem os registros dos resultados dos exames de triagem neonatal (teste do reflexo vermelho, teste de oximetria de pulso, triagem auditiva e avaliação do frênulo lingual). E como critérios de exclusão teve-se: prontuários de RN transferidos para outra unidade ou prontuários de RN que tiveram alta hospitalar antes da coleta de dados pelos pesquisadores.

Do total das 219 admissões, 103 prontuários foram excluídos do estudo pelos seguintes motivos: alta antes da coleta de dados nos prontuários (22); transferência para outro setor antes da coleta de dados (10) e sem os resultados de todos os exames de triagem neonatal (71). Portanto, a amostra foi composta por 116 prontuários.

A coleta de dados ocorreu em maio e junho de 2017 mediante o preenchimento de um instrumento de coleta composto pelas seguintes variáveis: dados maternos (realização de pré-natal e tipo de parto), dados do RN (medidas antropométricas, sexo, idade gestacional, idade do RN, tamanho do RN e apgar) e dados sobre os exames de triagem neonatal: teste do reflexo vermelho (presença ou ausência de leucocoria), oximetria de pulso (normal ou alterado), triagem auditiva (retorno de EOE presentes ou ausente) e avaliação do frênulo lingual (curto ou normal).

Os dados coletados na pesquisa foram armazenados em um banco de dados produzido pelo Excel do Windows 2010. Para a análise, utilizou a estatística descritiva, considerando frequências absolutas e relativas das variáveis, além de medidas de tendência central (média e desvio padrão (DP)). Os resultados foram apresentados em forma de tabelas.

Foram respeitados todos os preceitos éticos exigidos na resolução 466/12. Estudo aprovado pelo Comitê de Ética e Pesquisa da instituição via plataforma Brasil (CAAE 65931317.0.0000.5050).

\section{Resultados}

Os dados obstétricos maternos mostraram que 97,3\% das mães realizaram pré-natal, predominando a realização de pelo menos seis consultas $(81,9 \%)$, cuja quantidade de consultas variou de duas a 14 consultas com média de 7,6 e $\pm 2,42$. Os tipos de parto foram vaginal $(66,3 \%)$ e abdominal (33,7\%). Na tabela 1 tem-se o predomínio dos seguintes parâmetros antropométricos do RN: peso ao nascer dentro da normalidade (93,8\%), estatura entre $45 \mathrm{~cm}$ a $50,9 \mathrm{~cm}(86,4 \%)$, perímetro cefálico entre $32 \mathrm{~cm}$ a $35,9 \mathrm{~cm}(82,7 \%)$ e perímetro torácico entre $30 \mathrm{~cm}$ a $33,9 \mathrm{~cm}(56,0 \%)$.

Tabela 1. Caracterização das variáveis neonatais segundo as medidas antropométricas do recém-nascido

\begin{tabular}{lccc}
\hline $\begin{array}{l}\text { Variáveis neonatais } \\
\text { Medidas antropométricas }\end{array}$ & $\mathrm{n}(\%)$ & Média & DP \\
\hline $\begin{array}{l}\text { Peso (gramas) } \\
\quad 2.500 \mathrm{~g}\end{array}$ & $7(6,2)$ & 3151,8 & $\pm 425,97$ \\
$2.500 \mathrm{~g}-4.000 \mathrm{~g}$ & $106(93,8)$ & & \\
$\quad>4.000 \mathrm{~g}$ & $3(2,7)$ & & \\
Estatura $(\mathrm{cm})$ & & 48,5 & $\pm 1,99$ \\
43 a 44,9 & $3(2,5)$ & & \\
45 a 50,9 & $100(86,4)$ & & \\
51 a 54 & $13(11,1)$ & & \\
Perímetro cefálico $(\mathrm{cm})$ & & 34,4 & $\pm 1,31$ \\
30 a 31,9 & $3(2,5)$ & & \\
32 a 35,9 & $96(82,7)$ & & \\
36 a 38 & $17(14,8)$ & & \\
Perímetro torácico $(\mathrm{cm})$ & & 33 & \\
27 a 29,9 & $4(3,4)$ & & \\
30 a 33,9 & $65(56)$ & & \\
34 a 37 & $47(40,6)$ & & \\
\hline
\end{tabular}


Segundo a tabela 2, as seguintes características prevaleceram: sexo feminino $(42,2 \%), \mathrm{RN}$ nascidos a termo $(94,0 \%)$ e com tamanho adequado para a idade gestacional $(85,4 \%)$. O apgar prevalente obteve pontuação de oito a dez, com média de 8,2 pontos no primeiro minuto e 8,7 no quinto minuto.

Tabela 2. Caracterização das variáveis neonatais segundo sexo, idade gestacional, tamanho do RN, apgar e idade do RN

\begin{tabular}{|c|c|c|c|}
\hline Variáveis neonatais & $\mathrm{n}(\%)$ & Média & DP \\
\hline \multicolumn{4}{|l|}{ Sexo } \\
\hline Masculino & $49(42,2)$ & & \\
\hline Feminino & $67(57,8)$ & & \\
\hline \multicolumn{4}{|c|}{ Classificação idade gestacional } \\
\hline Pré-termo & $7(6)$ & & \\
\hline A termo & 109(94) & & \\
\hline \multicolumn{4}{|l|}{ Classificação tamanho x IG } \\
\hline PIG & $7(6)$ & & \\
\hline AIG & $99(85,4)$ & & \\
\hline GIG & $10(8,6)$ & & \\
\hline Apgar $1^{\circ}$ minuto de vida & & 8,2 & $\pm 0,976$ \\
\hline 3 a 4 & $2(1,7)$ & & \\
\hline 5 a 7 & $8(7)$ & & \\
\hline 8 a 10 & $104(89,6)$ & & \\
\hline Não registrado & $2(1,7)$ & & \\
\hline Apgar $5^{\circ}$ minuto & & 8,7 & $\pm 0,399$ \\
\hline 5 a 7 & $1(0,9)$ & & \\
\hline 8 a 10 & $112(96,4)$ & & \\
\hline Não registrado & $3(2,7)$ & & \\
\hline \multicolumn{4}{|l|}{ Idade do RN (horas) } \\
\hline Até $48 \mathrm{~h}$ de vida & $76(65,3)$ & & \\
\hline$>48 \mathrm{~h}$ de vida & $40(34,7)$ & & \\
\hline
\end{tabular}

Tabela 3. Dados sobre os exames de triagem neonatal nos RN

\begin{tabular}{l|c|c|c}
\hline Exames de triagem neonatal & $\mathrm{n}(\%)$ & Média & DP \\
\hline Teste do reflexo vermelho & & & \\
$\quad$ Leucocoria ausente & $115(99,2)$ & & \\
$\quad$ Leucocoria presente & $1(0,8)$ & & \\
Oximetria de pulso & & & \\
$\quad$ Sato2 membro superior direito (\%) & & 97,03 & $\pm 1,11$ \\
$\quad>95$ & $116(100)$ & & \\
$\quad$ Sato2 membros inferiores (\%) & & 96,9 & $\pm 1,004$ \\
$\quad>95$ & $116(100)$ & & \\
Triagem auditiva & & & \\
$\quad$ Retorno de EOE presente & $84(72,4)$ & & \\
$\quad$ Retorno de EOE ausente & $32(27,6)$ & & \\
Avaliação do frênulo lingual & & \\
$\quad$ Frênulo lingual normal & $108(93,2)$ & \\
$\quad$ Frênulo lingual curto & $8(6,8)$ & \\
\hline
\end{tabular}

No teste do reflexo vermelho, constatou-se que a maioria dos RN teve seus testes dentro das normalidades (leucocoria ausente) e um RN teve o teste alterado (leucocoria presente). Com relação aos dados maternos e neonatais do RN cujo exame dera alterado, tem-se: pré-natal realizado (oito consultas), parto normal, peso do RN dentro da normalidade (3435g), sexo masculino, $\mathrm{RN}$ nascido a termo, adequado para a idade gestacional, apgar sete no primeiro minuto e dez no segundo minuto e exames de triagem neonatal (teste de oximetria de pulso, triagem auditiva e avaliação do frênulo lingual) normais.

No teste da oximetria de pulso, este realizado pelas enfermeiras da unidade, a sat $\mathrm{O}_{2}$ prevalente no membro superior direito (MSD) e no membro inferior foi de $97 \%$, sendo que a satO2 no MSD variou de $95 \%$ a $99 \%$ e no membro inferior variou de $95 \%$ a $99 \%$.

De acordo com os registros feitos nos prontuários dos $\mathrm{RN}$, todos os testes de oximetria de pulso estavam dentro dos parâmetros da normalidade. No entanto, em dois RN, apesar dos registros constarem normalidade no resultado do exame, verificou-se que o mesmo dera alterado, pois um dos RN, o resultado da sato2 foi de $99 \%$ no MSD e $96 \%$ no membro inferior (diferença de $3 \%$ ), e em outro $\mathrm{RN}$ a sato2 foi de 98\% no MSD e $95 \%$ no membro inferior (diferença de $3 \%$ ). Esses achados evidenciam a necessidade de treinamento dos profissionais para correta interpretação dos resultados deste exame.

Na triagem auditiva, realizada pelo fonoaudiólogo, 72,4\% dos exames deram normais. Na avaliação do frênulo lingual, também realizado pelo fonoaudiólogo, 93,2\% dos exames estavam dentro dos parâmetros normais. Oito RN tiveram o exame alterado, pois apresentaram o frênulo lingual curto e todos eram do sexo masculino.

\section{Discussão}

A literatura referente à saúde ocular do $\mathrm{RN}$, com enfoque na detecção de problemas visuais, é bastante escassa e os conteúdos relacionados a essa temática ainda são pouco abordados pelos pesquisadores. ${ }^{(5)}$

Neste estudo, a maioria dos $\mathrm{RN}$ teve o teste do reflexo vermelho dentro da normalidade. Apenas um $\mathrm{RN}$ teve o teste alterado, sendo encaminhado para avaliação com o oftalmologista da instituição.

Achados parecidos foram encontrados em um estudo realizado com $\mathrm{RN}$ admitidos em uma maternidade de Santa Catarina, na qual constatou que dos 10.135 RN submetidos ao teste do reflexo vermelho, 99,97\% 
apresentaram parâmetros dentro do esperado e 0,03\% apresentaram alterações no exame, sendo que um era alteração bilateral e dois unilateral e todos foram encaminhados para serviços de referência em oftalmologia pediátrica. ${ }^{(6)}$

Outro estudo realizado em uma maternidade de Fortaleza-CE, com 190 RN, evidenciou que 98,42\% tiveram o exame do reflexo vermelho normal, e os $\mathrm{RN}$ cujo exame dera alterado foram incluídos no programa de rastreamento e tratamento da retinopatia da prematuridade da instituição. ${ }^{(5)}$

O resultado do exame de oximetria de pulso realizados nos $\mathrm{RN}$ foram todos registrados nos prontuários como normais (sato2 > 95\%) (100\%). Entretanto, dois RN apresentaram alterações nos exames, pois houve diferença de 3\% entre a sato2 dos MSD e membros inferiores, indicando a possibilidade de alguma cardiopatia. Vale ressaltar que alterações no teste de oximetria de pulso pode ser indicativo de cardiopatia congênita, portanto destaca-se a importância da realização correta do exame para que se possa garantir uma assistência adequada a esses RN.

Perante o que foi exposto acima, pesquisa realizada com 13 enfermeiras neonatologistas de uma maternidade da Paraíba, evidenciou que a maioria $(85 \%)$ das enfermeiras entrevistadas sabia identificar os parâmetros de normalidades do exame de oximetria de pulso de acordo com as normas propostas pelo Ministério da Saúde. ${ }^{(7)}$

Na instituição estudada, RN com exames de oximetria de pulso alterados são encaminhados para realização de ecocardiograma com um cardiologista para confirmar ou descartar algum diagnóstico de cardiopatia congênita.

Estudo realizado com 4.027 RN, para avaliação dos exames de oximetria de pulso, constatou que $0,5 \%$ dos testes deram alterados, e todos os RN foram encaminhados para realização do ecocardiograma, na qual se confirmou cardiopatias congênitas como comunicação interventricular (CIV) em quatro RN; drenagem anômala total das veias pulmonares (DATVP) em um RN e transposição das grandes artérias (TGA) em um RN. ${ }^{\left({ }^{(8)}\right.}$

Valores maiores foram encontrados em um estudo realizado com $\mathrm{RN}$ internados em unidades de terapia intensiva de um hospital da China, na qual identificou-se que dos $4.128 \mathrm{RN}$ que realizaram o teste de oximetria de pulso, 55,9\% tiveram o exame alterado. ${ }^{(13)}$
Quanto ao exame de triagem auditiva neonatal, constatou-se que $27,6 \%$ deram alterados. Os RN cujos exames estavam fora dos parâmetros da normalidade, foram encaminhados para o serviço especializado na própria instituição, para realização do teste novamente após sete dias para confirmação ou descarte da alteração.

Ressalta-se que boa parte dos exames de triagem auditiva alterados são geralmente ocasionados por presença de líquido do parto presente no ducto auditivo do RN, sendo que a maioria dos retestes feitos no serviço descartam alterações na audição.

Estudo conduzido em Belo Horizonte com prontuários de 4.442 crianças atendidas em um serviço de triagem auditiva, constatou que $64,3 \%$ delas realizaram o exame nos primeiros 30 dias de vida e que $7,3 \%$ apresentaram alterações no exame, sendo encaminhadas para o reteste com o fonoaudiólogo. ${ }^{(14)}$

Outro estudo realizado com 313 prontuários de crianças atendidas em uma unidade de reabilitação constatou que $34 \%$ das crianças tiveram o exame de triagem auditiva neonatal alterado, as quais iniciaram o tratamento antes dos seis meses de idade, conforme o recomendado pelo Comitê Multiprofissional em Saúde Auditiva (COMUSA) e pelas Diretrizes de Atenção para a Triagem Auditiva Neonatal. Ainda no mesmo estudo, a maioria das crianças $(92,9 \%)$ apresentou alteração auditiva bilateral. ${ }^{(10)}$

Em outra pesquisa realizada em uma unidade de terapia intensiva neonatal, pode-se evidenciar que dos 140 prontuários estudados, a idade média de realização da triagem auditiva neonatal foi de 66 dias, sendo que $11,42 \%$ tiveram o teste alterado. ${ }^{(15)}$

Alterações no frênulo lingual e sua interferência na amamentação têm sido relatadas em várias pesquisas. Diante disso, a avaliação do frênulo lingual em RN identifica a necessidade ou não de frenotomia, sendo este procedimento necessário quando detectado o encurtamento do frênulo lingual no bebê..$^{(11)}$

Na avaliação do frênulo lingual dos $\mathrm{RN}$, constatou-se que a maioria não necessitava de frenotomia. Sendo que os RN cujos exames deram alterados foram encaminhados para realização de frenotomia com o cirurgião pediátrico da instituição.

Pesquisa realizada em um hospital maternidade no estado de São Paulo evidenciou que dos 100 RN submetidos à avaliação do frênulo lingual, $21 \%$ dos 
exames deram alterados, no entanto $36,3 \%$ desse percentual estavam sendo amamentados e fazendo uso de fórmulas artificiais para complementar a dieta. ${ }^{(11)}$

Uma das limitações do estudo foi a não avaliação dos resultados do teste do pezinho haja vista que este só pode ser coletado após $72 \mathrm{~h}$ de vida. No entanto, durante a coleta de dados, evidenciou-se que dos 116 prontuários pesquisados, $44 \mathrm{RN}$ haviam realizado o teste do pezinho, ou seja, os mesmos estavam internados na instituição havia mais de 72 horas, contudo os pesquisadores não tiveram acesso ao resultado exame por este ser liberado pelo laboratório somente com 45 dias.

Outra limitação do estudo refere-se à população estabelecida na pesquisa, na qual incluiu-se RN admitidos no setor somente durante o mês de maio do ano de 2017.

\section{Conclusão}

Pode-se concluir com os achados desta pesquisa que maioria dos $\mathrm{RN}$ submetidos aos exames de triagem neonatal teve seus resultados dentro da normalidade, sendo estes exames, o teste do reflexo vermelho, o teste de oximetria de pulso, a triagem auditiva e a avaliação do frênulo lingual. Os RN cujos exames deram alterados foram encaminhados para serviços especializados para resolução do problema ou acompanhamento ambulatorial. Constatou-se também a necessidade de treinamento/capacitação da equipe de enfermeiros no que diz respeito ao teste de oximetria de pulso, tendo em vista o registro incorreto do resultado do exame em dois prontuários de $\mathrm{RN}$, ocultando assim a existência de uma possível cardiopatia no RN. Por fim, destaca-se a baixa produção científica encontrada sobre os exames de triagem neonatal, tanto na literatura nacional como internacional, como o teste do reflexo vermelho e o teste de oximetria de pulso, denotando a necessidade de mais pesquisas sobre o assunto.

\section{Colaborações}

Mendonça LBA, Lima FET, Freitas I, Gurgel SS, Ferreira MKM e Sandoval LJS declararam que contribuíram com a concepção do estudo, análise e interpretação dos dados, redação do artigo, revisão crítica relevante do conteúdo intelectual e aprovação da versão final a ser publicada.

\section{Referências}

1. Mesquita AP, Marqui AB, Silva-Grecco RL, Balarin MA. Profissionais de Unidades Básicas de Saúde sobre a triagem neonatal. Rev Ciênc Méd. 2017;26(1):1-7.

2. Marqui AB. The blood drop screening (guthrie) test and the role of nurses: a reflection. Rev Enferm Atenção Saúde .2016;5(2):96-103.

3. Calvo-Gonzalez E. Biotecnologias de baixa complexidade e aspectos cotidianos do "cuidado": a triagem neonatal e a detecção da doença falciforme no Brasil. Hist Cienc Saude-Manguinhos. 2016;23(1):79-94.

4. Arduini GA, Balarin MA, Silva-Grecco RL, Marqui AB. Knowledge of puerperal mothers about the guthrie test. Rev Paul Pediatr. 2017;35(2):151-7.

5. Aguiar AS, Ximenes LB, Lúcio IM, Pagliuca LM, Cardoso MV. Associação do reflexo vermelho em recém-nascidos com variáveis neonatais. Rev Lat Am Enfermagem. 2011;19(2):309-16.

6. Cagliari PZ, Silva JC, Veras TN, Vieira CE, Bertelli U, Ramos MC. Alterações detectadas pelo teste do reflexo vermelho. Arq Catarin Med. 2016;45(3):48-57.

7. Medeiros AL, Freitas TB,Araújo JS, Mattos SS. Oximetria de pulso em triagem de cardiopatias congênitas: conhecimento e atuação do enfermeiro. Cogitare Enferm. 2015;20(3):601-7.

8. Albuquerque FC, Maia ET, Figueiredo VL, Mourato FA, Mattos SS. Exame Físico e Oximetria de Pulso para Detectar Cardiopatias congênitas. Int J Cardiovasc Sci. 2015;28(2):14851.

9. Costa AP, Raignieri FS, Figueiredo KJ, Espinosa MM, Nardez TM, Rodrigues PA. Avaliação do programa de triagem auditiva neonatal da Clínica Escola do Univag. Rev CEFAC. 2016;18(2):335-40.

10. Rodrigues GR, Loiola-Barreiro CM, Pereira T, Pomilio MC. A triagem auditiva neonatal antecipa o diagnóstico e a intervenção em crianças com perda auditiva? Audiol Commun Res. 2015;20(3):246-54.

11. Martinelli RL, Marchesan IQ, Lauris JR, Honório HM, Gusmão RJ, Berretin-Felix G. Validade e confiabilidade da triagem: "teste da linguinha". Rev CEFAC. 2016;18(6):1323-31.

12. Strefling IS, Monfrim XM, Lunardi Filho WD, Carvalho KK, Azevedo AL. Conhecimento sobre triagem neonatal e sua operacionalização. Cogitare Enferm. 2014;19(1):27-33.

13. Hu XJ, Zhao QM, Ma XJ, Yan WL, Ge XL, Jia B, Liu F, et al. Pulse oximetry could significantly enhance the early detection of critical congenital heart disease in neonatal intensive care units. Acta Paediatr. 2016; 105(11):e499-e505.

14. Januário GC,Alves CR, Lemos SM, Almeida MC, Cruz RC, Friche AA. Índice de Vulnerabilidade à Saúde e triagem auditiva neonatal: diferenciais intraurbanos. CoDAS. 2016; 28(5):56774.

15. Rechia IC, Liberalesso KP, Angst OV, Mahl FD, Garcia MV, Biaggio EP. Intensive care unit: results of the Newborn Hearing Screening. Braz J Otorhinolaryngol. 2016;82(1):76-81. 